\title{
Summary and Recommendations of the Expert Panel Oversight Committee Meeting on Double- Shell Tank Corrosion Monitoring and Testing Held August 4 and 5, 2008
}

\section{K. D. Boomer}

WRPS

Richland, WA 99352

U.S. Department of Energy Contract DE-AC27-08RV14800

\begin{tabular}{|c|c|c|c|}
\hline EDT/ECN: & DRF & UC: $\quad$ N/A & \\
\hline Cost Center: & $\mathrm{N} / \mathrm{A}$ & Charge Code: & N/A \\
\hline \&R Code: & N/A & Total Pages: & 15 f 15 \\
\hline
\end{tabular}

Key Words: EPOC, Double Shell Tank Corrosion, Monitoing \& Testing, CC Technologies,

Abstract: The Expert Panel Oversight Committee (EPOC) on Double-Shell Tank Corrosion Monitoring and Testing has been overseeing the Fiscal Year (FY 2008 experimental program being performed at CC Technologies (CCT) to optimize the chemistry control for corrosion limits in Double-Shell Tanks (DSTs). The EPOC met at the M \& D Professional Services Conference Facility on August 4 and 5, 2008 to discuss various aspects of that responsibility including FY 2009 planning. Formal presentations were made to update the EPOC on the these subjects

TRADEMARK DISCLAIMER. Reference herein to any specific commercial product, process, or service by trade name trademark, manufacturer, or otherwise, does not necessarily constitute or imply its endorsement, recommendation, or favoring by the United States Government or any agency thereof or its contractors or subcontractors.
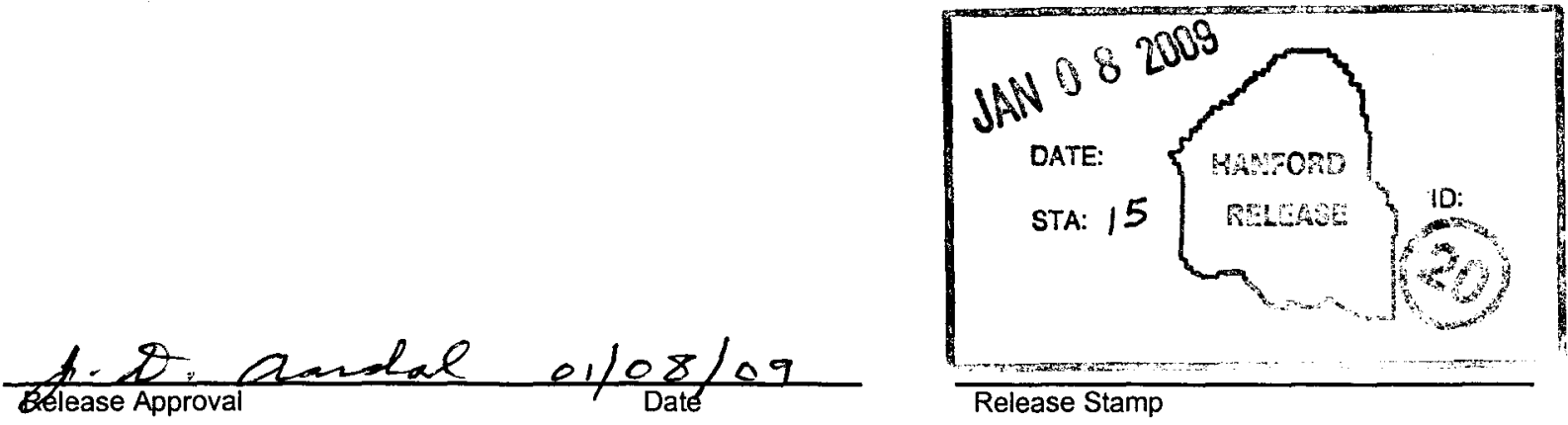

\section{Approved For Public Release}


MICHAEL T. TERRY, P.E.

323 Skagit St.

Richland, Washington 99354

(509) 531-0920

September 12, 2008

Mr. Herbert Berman

CH2M HILL, Hanford Group Inc.

P.O. Box 1500, MSIN R2-58

Richland, Washington 99352

\title{
SUBJECT:
}

\author{
SUMMARY AND RECOMMENDATIONS OF THE EXPERT \\ PANEL OVERSIGHT COMMITTEE MEETING ON DOUBLE- \\ SHELL TANK CORROSION MONITORING AND TESTING \\ HELD AUGUST 4 AND 5, 2008
}

Dear Mr. Berman,

The Expert Panel Oversight Committee (EPOC) on Double-Shell Tank Corrosion Monitoring and Testing has been overseeing the Fiscal Year (FY 2008 experimental program being performed at CC Technologies (CCT) to optimize the chemistry control for corrosion limits in Double-Shell Tanks (DSTs). The EPOC met at the M \& D Professional Services Conference Facility on August 4 and 5, 2008 to discuss various aspects of that responsibility including FY 2009 planning.

Formal presentations were made to update the EPOC on the following subjects:

- Defense Nuclear Facilities Safety Board Update/Hanford Update

- Corrosion and Stress Corrosion Cracking (SCC) Testing of Hanford Site Wastes at CCT

- Hanford DST 241-AN-102 Multi-probe Corrosion Monitoring System (MPCMS) Corrosion Monitoring Overview

- Chemical Thermodynamic Modeling for Vapor Space Corrosion Testing

- Savannah River Site - Vapor Space Corrosion Testing Update

- EM-21 Tank Storage FY 2009 Updated Proposed Activities

- Fracture Mechanics Testing Program 2009

Following these presentations, the EPOC identified several topics for further discussion. Attachment 1 provides summaries of each of the areas discussed as well as recommendations associated with each topic.

The EPOC has concluded the testing program for the interstitial liquids in the present and future solid layers of the DSTs is complete. The results of this test program can be used to modify the chemistry limits for the interstitial liquids. We further conclude that the testing program for pitting corrosion and SCC of the present liquid supernatant layers of the DST can be completed

Double-Shell Tank Corrosion Monitoring and Testing Expert Panel Oversight Committee Members

$$
\begin{gathered}
\text { John Beavers • Gerald Frankel • Leon Stock } \\
\text { Michael Terry } • \text { Bruce Wiersma }
\end{gathered}
$$


by carrying out the short test program laid out in the attachment. Additional pitting corrosion and SCC testing is required in order to establish the chemistry limits for tanks that will be used to receive waste retrieved from the Single-Shell Tanks.

The goals for testing for FY 2009 have been proposed (see attachment), which will lead to the establishment of new chemistry limits for all of the DSTs.

We continue to be pleased that CH2M HILL is taking such a responsible and thorough approach to evaluating and implementing the recommendations of the EPOC.

Sincerely,

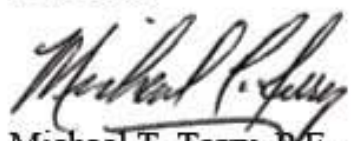

MichaeT T. Terry, P.

Chairman, Double-Shell Tank Corrosion

Monitoring and Testing Expert Panel Oversight

Committee 


\section{Attachment 1 \\ Summaries of Discussion Topics from the August 4 and 5, 2008 EPOC Meeting}

This meeting was held to update the EPOC on the status of the tank waste corrosion testing being conducted at CC Technologies, Inc and the in-tank corrosion monitoring probe development being performed by ARES Corporation CH2M HLL instituted these activities in response to recommendations in the full Chemistry Optimization Panel report, RPP-RPT-22126, Expert Panel Workshop for Honford Site Double-Shell Tank Waste Chemistry Optimization, October 2004. Specifically, these programs addressed recommendations related to Initiative I, DoubleShell Tank Operation Outside Established Chemistry Control Specifications for Limited Periods of Time, and Initiative III, Corrosion Chemistry Specification Modifications,

\subsection{FY 200S TESTING PROGRAM CLOSEOUT}

With only two slow strain rate (SSR) tests remaining to be performed this fiscal year, the Panel reviewed the summary chart presented during the Corrosion and SCC Testing presentation (see Figure 1), and following a short discussion, focused on the need for additional information in the area around $-100 \mathrm{mV}$ (SCE) using a modified AW105-PSC (Present Supematant Composition) with only half the nitrite composition of the base AW105-PSC test solution as used in Test ID number 73. The concentrations of both nitrite and nitrate ions for the last two tests are to be increased by multiples of six and three, respectively.

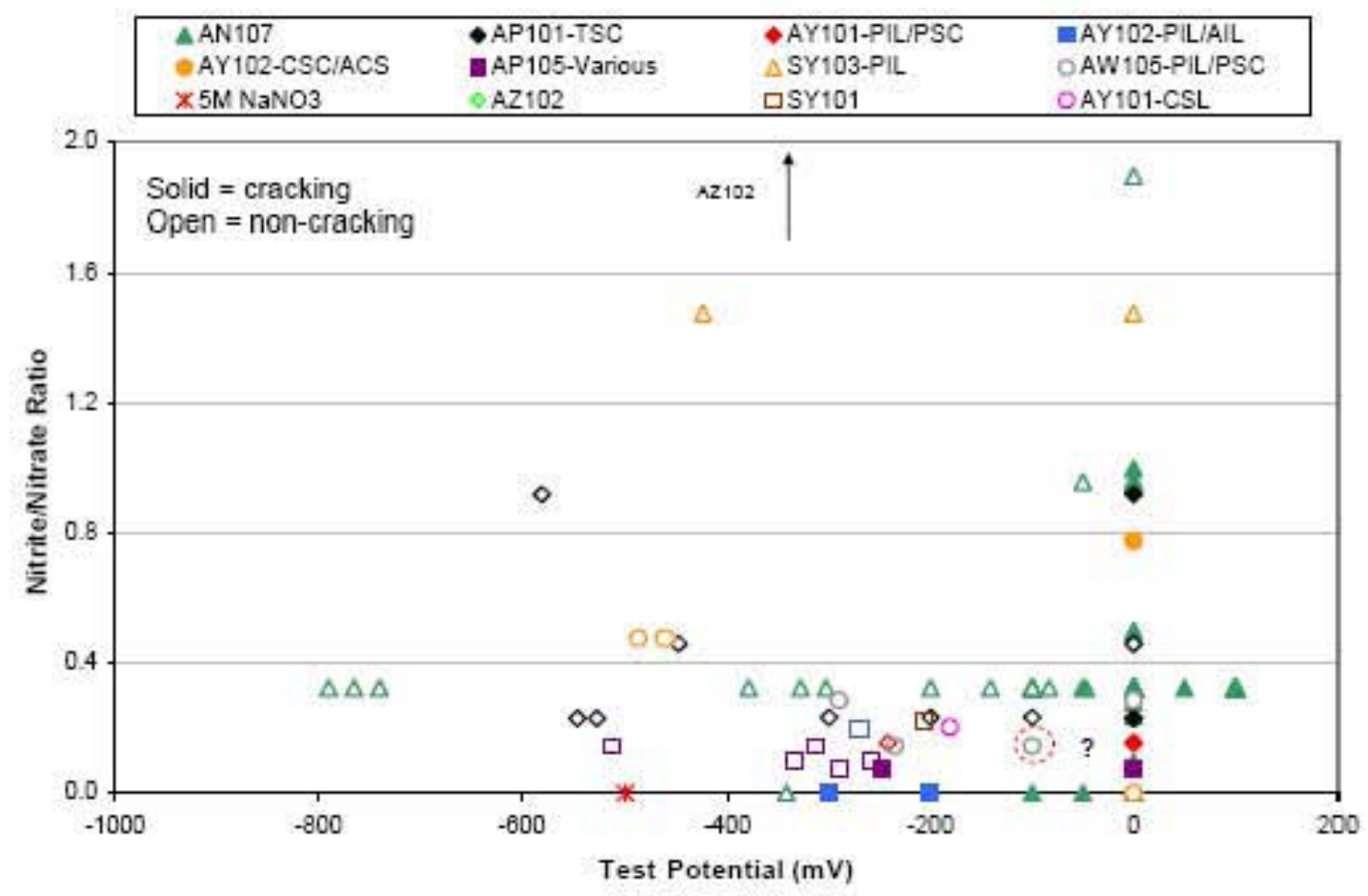

Figure 1. Flot of nitrite/ritrate ratio vs. potential for various SSRT data. 
We conclude that the testing program for the interstitial liquids in the present and future solid layers of the Double-Shell Tanks (DSTs) is complete. We further conclude that the testing program for pitting corrosion and SCC of the present liquid supernatant layers of the DST can be completed by carrying out the short test program outlined in Section 2. Additional pitting corrosion and SCC testing is required in order to establish the chemistry limits for tanks that will be used in retrieval operations.

Information presented at the meeting indicates that the compositions of the wastes in the DSTs will be altered significantly in the future as a result of the transfer of wastes from the SSTs to the DSTs. A testing program to investigate this matter is outlined in Section 2. Any work that is done in this area should be closely coordinated with the results obtained during the testing of the supernatant layers and the interstitial liquids from the solid layers of the DSTs and future testing programs of the SSTs.

An additional area of concern is corrosion at the liquid-air-steel interface. We recommend the testing program outlined in Section 2

\section{EPOC Recommendations}

1. Conduct the first of the remaining two FY 2008 SSR tests at CCT using a modified test solution consisting of the chemical composition from Test ID 73 (AW105-PSC with half nitrite composition) further modified by multiplying the nitrite and nitrate ion concentrations by six times. The second test will use the same Test ID 73 solution, however, nitrite and nitrate concentrations will be multiplied by 3 .

It should be noted that subsequent to the workshop, test (ID 74) was performed with the same nitrite to nitrate ratio, however, the nitrate and nitrite concentrations were multiplied six-fold, as recommended. Since no SCC was evident, and after consultation with the EPOC, CCT initiated the final test with the nitrate and nitrite concentrations from Test 74 and with an applied potential of $-50 \mathrm{mV}$.

\subsection{FY 2009 TESTING PROGRAM}

We believe that more work is required to complete the understanding of the propensity for pitting and SCC corrosion in the existing supernatant layers of the DSTs. Several issues remain as detailed below.

\section{Effects of Temperature, Ammonia, and Pertechnetate}

Previous studies in several laboratories imply that temperature is an important factor in nitrate ion induced SCC. Specifically, slow strain rate (SSR) and related tests in several laboratories that were carried out with nitrate ion rich simulants of Hanford Site wastes caused SCC at temperatures greater than $50^{\circ} \mathrm{C}$, but the tests that were carried out at lower temperatures $(25$ and $40^{\circ} \mathrm{C}$ ) did not cause SCC. Inasmuch as the SSR tests at CCT have been carried out conservatively at $50^{\circ} \mathrm{C}$, we recommend that several SSR tests be performed to clarify the effect of temperature on the propensity of SCC for the supernatant DST wastes, and to assess more definitely the conservatism of the present testing program.

Early laboratory and fieldwork at the Hanford Site led to the suggestion that ammonia, which is a ubiquitous constituent of these wastes, may inhibit corrosion in the liquid and vapor phases. This 
proposal has not been independently investigated. Accordingly, we recommend that exploratory work be carried out to define the propensity for pitting corrosion and SCC in the presence of ammonia for the supernatant DST wastes by using CPP and SSRT.

The suggestion has been advanced that pertechnetates in the Hanford Site wastes inhibit corrosion, for example in Tank 241-AN-107. We recommend that the technical literature regarding the notion that pertechnetates inhibit corrosion be reviewed. We also recommend that the waste compositions be reviewed to determine the amounts of pertechnetates in the Hanford Site waste tanks. If evidence is found for inhibiting effects of pertechnetates, and their concentration in wastes is reasonably high, experiments should be performed to investigate their effects in waste simulants. These experiments must be performed in a hot cell.

\section{EPOC Recommendations}

1. SSR tests be performed to clarify the effect of temperature on the propensity of SCC for the supernatant DST wastes, and to assess more definitely the conservatism of the present testing program.

2. Exploratory experiments on the effects of ammonia

3. Review of literature on effects of pertechnetates, followed by hot cell experiments if warranted.

\section{Determination of critical potential and concentrations}

A full understanding of the region of corrosion and cracking susceptibility and the margins relative to operating conditions is needed for the EPOC to recommend changes in the supernatant chemistry limits. Ongoing experiments have shown that corrosion and cracking are dependent on a large number of factors such as nitrate, nitrite, hydroxide, potential, carbonate, aluminate, chloride, ammonia and temperature. Various effects have been studied using a "linear" approach of changing one parameter while holding the others at set values. In particular, the region at low nitrite/nitrate ratio and potential in the range of -100 to $0 \mathrm{mV} \mathrm{SCE}$ has been examined in some detail. However, for most of those experiments, the other parameters were all kept constant.

This linear approach provides insight, but does not allow for complete examination of the experimental space or understanding of interactions between parameters. One way to study a wider range of parameter values is to use a statistical design of experiments approach such as factorial experiments in which the parameters or factors are varied with discrete values or levels. Fractional factorial experiments allow for some subset of the possible combination of values to be investigated.

\section{$\underline{\text { EPOC Recommendation }}$}

1. A statistical experimental design approach is recommended to cover the factor space with a reasonable number of tests. The model should be developed that predicts the probability of cracking as a function of nitrate, nitrite, hydroxide, potential, carbonate, aluminate, chloride, ammonia and temperature.

\section{Liquid/Air Interface}

Concerns about corrosion at the supernatant liquid/air interface (LAI) and in the vapor space above the supernate are preventing implementation of new chemistry controls with lower $\mathrm{pH}$ 
limits for the supernatant layers that have been implemented for the interstitial liquid in the sludge layers. Experiments by CCT have shown that severe LAI corrosion can occur for the 241-AP-105 simulant under certain conditions, even though the $\mathrm{pH}$ was in excess of 13 . In particular, high potential ( $0 \mathrm{~V} \mathrm{SCE}$ compared to OCP), high temperature $\left(50\right.$ compared to $\left.25^{\circ} \mathrm{C}\right)$, the presence of oxygen and $\mathrm{CO}_{2}$ in the air space, and stagnant exposure (as opposed to active sparging, which results in mixing) were found to promote LAI corrosion. Evidence of LAI corrosion has also been generated by DOE sponsored work in Argentina and at SRNL. As with other testing, the nitrite showed a greater inhibition of LAI corrosion than did hydroxide, but LAI corrosion occurred in test regions where bulk solutions showed no propensity for pitting corrosion or SCC. It is clear to the EPOC that an improved understanding of LAI corrosion is necessary.

The EPOC recommends that a two-phase approach be taken to study LAI corrosion. In phase 1, a few parameters suggested by the technical literature to be possible strong effects in the mitigation of LAI corrosion will be studied. These parameters include nitrite, nitrite to nitrate ratio, $\mathrm{pH}$, temperature, steel microstructure and surface condition, and ammonia. It is hoped that the results of these experiments will provide considerable insight into the susceptibility of Hanford tanks to LAI corrosion. However, it is likely that the development of chemistry control specifications for the prevention of LAI corrosion will require a more detailed study, given the number of parameters involved. The details of this second phase of work will depend on the results of Phase 1.

Experiments to investigate LAI corrosion should be performed on partially immersed samples exposed to simulants with marginal corrosivity like 241-AP-105 and with a range of gas control. The important parameters appear to be:

1. Chemistry: various nitrite/nitrate ratios and absolute concentrations, hydroxide

2. Gas: air, zero air $\left(\mathrm{N}_{2}+\mathrm{O}_{2}\right), \mathrm{O}_{2}$-free $\left(\mathrm{N}_{2}\right.$ or $\left.\mathrm{Ar}\right)$, ammonia

3. Temperature: Room Temperature to $50^{\circ} \mathrm{C}$

4. Potential: OCP to $0 \mathrm{~V} \mathrm{SCE}$

5. Surface condition of metal: polished, mill-scale, etc.

Different tests could be performed:

1. Potentiodynamic polarization - to get a quick assessment of corrosivity.

2. Potentiostatic testing - performed at a high potential such as $0 \mathrm{~V}$ SCE. The current can be measured as a function of time and conditions changed to see critical concentrations for initiation or inhibition of attack. For instance, it is possible to titrate nitrite into an aggressive solution to determine the nitrite needed to inhibit attack. Care must be taken to avoid agitation when adding solution to adjust the concentration as mixing of bulk solution with the LAI can inhibit attack. The addition must be slow and time given for mixing by diffusion and natural convection. If a successful protocol can be established, limits of nitrite, nitrite/nitrate, or hydroxide for avoiding LAI corrosion might be determined.

3. Long term OCP exposures to a range of conditions - to investigate stability at low potentials. 
Analytical methods such as Raman Spectroscopy will be needed to sort out the fundamental mechanism of LAI corrosion including the changes in chemistry at the LAI that are responsible for the accelerated attack. However, this work is better suited for a university-based program.

\section{$\underline{\text { EPOC Recommendation }}$}

1. LAI corrosion should be studied using a phased approach. The effects of temperature, steel microstructure and surface condition, and ammonia should be addressed first to determine if any of these are strongly mitigating factors. To develop new chemistry specifications, a more detailed study should follow.

\section{Vapor Space Corrosion}

The overall goals of the research on vapor space corrosion (VSC) include establishing the potential threat of VSC for the existing conditions in the DSTs and the conditions planned for waste retrieval operations and evaluating the potential effect of changes in the corrosion control specification on VSC. While some recent test results from SRNL suggest that changing from hydroxide to nitrite control will not measurably affect VSC, additional testing will be required to substantiate this conclusion. VSC corrosion issues and recommended testing are discussed below.

\section{Chemical Modeling}

Complex mixtures are formed as a consequence of the interactions that occur between solids, liquids, and gases on the walls in the headspaces of the DST. The EPOC previously recommended that modeling work be performed to determine the thermodynamic composition of these potentially corrosive mixtures. Felmy and Qafoku presented the results of their recent investigation of the compositions of the mixtures that result from the drying of condensate solutions, the thermodynamics of the removal of water from the supernatant liquid of Tank 241-AZ-102 (PUREX aging waste type), the compositions of the dilute condensates that collected in the AZ-301 Seal Pot, the thermodynamic relationship between ammonia and carbon dioxide in the headspaces and in condensates at different $\mathrm{pH}$, and the relationship between the hydroxide content of the supernatant layer and the carbon dioxide content of the headspace. The EPOC recommends that additional work be carried out on the consequences of drying condensates and supernatant waste solutions as outlined in the next two sub-sections.

\section{Consequences of Drying Condensates}

Investigations of the compositions of the mixtures that result from the drying of condensate solutions were begun by Felmy and Qafoku in FY 2008. They examined the thermodynamic relationships between the constituents found in condensates of the AZ-301 Seal Pot as water evaporated. They determined the changes in $\mathrm{pH}$ and the conditions that led to the precipitation of solids from the solutions. In brief, they found aluminum hydroxide and calcium carbonate precipitated from solution when only modest amounts of water were evaporated, but the other constituents including ammonium nitrate, ammonium bicarbonate, ammonium carbamate, ammonium sulfate, and ammonium chloride remained in these solutions until virtually all the water has been removed. They also found that about $90 \%$ of the water needed to be removed from the dilute condensate solutions before the $\mathrm{pH}$ changed perceptibly. It is not clear what factors govern the direction of the observed $\mathrm{pH}$ change, but the thermodynamically predicted ending $\mathrm{pH}$ was about 10 when the initial $\mathrm{pH}$ was about 9.3 or 9.8 , and the ending $\mathrm{pH}$ was about 8.4 when the original $\mathrm{pH}$ was 8.8 . 
The thermodynamic modeling indicates that ammonia and carbon dioxide produce carbamate ion in these concentrated liquid solutions. The thermodynamic evaluation also indicates that ammonium carbamate eventually precipitates from these solutions together with ammonium bicarbonate and ammonium nitrate. The new thermodynamic computations clearly indicate that simulants for solutions that form by the evaporation of dilute condensate solutions, which initially contain ammonium nitrate and ammonium carbonate, should contain nitrate ion, ammonia, ammonium ion, carbamate ion, bicarbonate and carbonate ion.

This very fruitful investigation at PNNL suggests that ammonium carbamate and ammonium bicarbonate precipitate from solution together with sodium carbonate when the solution evaporates. However, analyses of the solid materials collected in the AZ-702 area do not contain inorganic carbon. At the present level of understanding, these two findings appear to be incompatible. However, the technical literature indicates that a one-to-one solid mixture of ammonium bicarbonate and ammonium carbamate spontaneously evolves ammonia and carbon dioxide in air.

\section{$\underline{\text { EPOC Recommendations }}$}

1. First, it is recommended that the thermodynamic findings that have already been obtained be verified experimentally.

2. Second, the condensates contain other substances that have not yet been included in the thermodynamic modeling and they may contain other unsuspected substances, for example carboxylic acids and urea that have not yet been definitely identified analytically or considered in the thermodynamic work. It is appropriate to investigate whether urea is a plausible constituent and whether carboxylic acids can alter the thermodynamic relationships. Consequently, it is recommended that additional modeling work be done. One avenue that presumably needs to be explored concerns the influence of small concentrations of acids, for example acetic acid, on the thermodynamic outcome.

\section{Consequences of Drying Supernatant Solutions}

The compositions of the mixtures that result from the drying of supernatant solutions need to be determined so that simulants can be formulated for corrosion testing. The liquids that result from the evaporation of water have high ionic strength and therefore their compositions need to be examined by analytical tests or by advanced thermodynamic methods or by both approaches.

Felmy and Qafoku investigated the thermodynamics of the removal of water from the supernatant liquid of Tank 241-AZ-102 (PUREX aging waste type). The evaporation of water from this waste led to the early precipitation of sodium sulfate-sodium fluoride, calcium hydroxide, and sodium carbonate monohydrate. Sodium nitrate began to precipitate after about $80 \%$ of the water had been removed. Anhydrous sodium carbonate precipitated after the removal of about $90 \%$ of water. The solution became increasingly alkaline as the water was removed with $\mathrm{pH}$ values greater than 16. There was no indication in the preliminary report that sodium nitrite precipitated from solution. The inference of the available thermodynamic calculations is that the concentrated solution achieved by the evaporation of water contained increasingly higher concentrations of sodium hydroxide and sodium nitrite. The thermodynamically predicted composition of a solution that formed from a completely solid form of this waste by the addition of a small amount of water would presumable contain the same constituents. The thermodynamic work that has been carried with the Tank 241-AZ-102 waste implies that the nitrite ion/nitrate ion concentration ratio and the hydroxide ion content change appreciably when the water content is lowered or 
raised, i.e. in the last stages of evaporation or the first stages of dissolution. These changes could have a very important impact on corrosion.

\section{EPOC Recommendations}

1. Additional thermodynamic modeling of the drying of supernatant wastes needs to be carried out. It is recommended that the modeling work be extended to include at least three additional waste types from the group noted in RPT 13639, Caustic Limits Report -May 2007, to gain a better understanding of the possible compositional variations.

2. Second, it is recommended that the thermodynamic predictions that have already been reported be verified by the experimental investigation of the compositions of solutions formed from waste simulants by the evaporation of water and independently by the addition of water to the same solids.

\section{Stress Intensity Factor and Crack Growth Rate Testing}

The overall goal of the fracture mechanics tests was to provide an additional level of conservatism in the optimization of the chemistry control for the DSTs. This goal can be accomplished by demonstrating that the maximum applied stress intensity factor $\left(\mathrm{K}_{\text {applied }}\right)$ at the highest stress region of a tank is below the threshold $\mathrm{K}$ for SCC initiation and growth $\left(\mathrm{K}_{\mathrm{thSCC}}\right)$ for the different waste chemistries. $K_{\text {applied }}$ can be calculated based on the maximum flaw size and applied stress that could be present in the tank There are several test techniques that can been used to estimate $\mathrm{K}_{\mathrm{thSCC}}$ and the technique(s) have evolved in this program. All have utilized a pre-cracked compact type specimen geometry, which is well characterized with respect to the K vs. crack length relationship and crack length calculations based on electric potential drop (EPD). These techniques were the subject of discussion at the EPOC meeting.

Three basic test techniques have been used in the DST research program to estimate $\mathrm{K}_{\text {thSCC}}$; constant load, constant displacement rate, and constant displacement rate followed by constant displacement. The modifications to the tests techniques were made in response to technical issues identified in the program, including specimen-to-specimen variability in the test results, poor sensitivity to crack initiation with the EPD measurements, and the potential for displacement rate effects on $\mathrm{K}_{\text {thSCC. }}$.

A relatively small number of tests have been performed with the newest technique (constant displacement rate followed by constant displacement), with no replicates under the same test conditions. There are some discrepancies in the few cases where the results can be compared with previous constant load or constant displacement rate results. For example, in Test CT-13, the $\mathrm{K}_{\text {thsCC }}$ was estimated to be around $35 \mathrm{ksi} \sqrt{ }$ in the $\mathrm{AN}-107$ chemistry at $0 \mathrm{mV}$ polarized potential and $50{ }^{\circ} \mathrm{C}$; whereas intergranular crack growth was reported in one constant load test at a much lower applied $\mathrm{K}(10 \mathrm{ksi} \sqrt{ } \mathrm{in})$ but otherwise, under the same test conditions. It should be pointed out that, in the early tests, the presence of intergranular SCC was established based on metallography and was not confirmed by means of an SEM examination; the analysis might have been in error.

Two other issues associated with the fracture mechanics tests were discussed in the EPOC meeting; the testing of welded samples and the state of stress. The majority of the failures in the single shell tanks have been associated with welds. The general consensus is that the primary function of the welds is to produce high residual tensile stresses, based on the fact that cracking also has occurred in the heat affected zone (HAZ) and base metal near the welds. However, the relative SCC susceptibility of the different microstructures associated with the welds (weld metal, 
coarse grain $\mathrm{HAZ}$, fine grain $\mathrm{HAZ}$, etc) has not been investigated for the various simulated wastes.

The state of stress refers to the level of constraint at the crack tip in the fracture mechanics test. In standard $\mathrm{K}_{\mathrm{IC}}$ testing it is desirable to have a high level of constraint in the test specimen in order to have valid, conservative results. All other things being equal, a thinner specimen, which has less constraint, will typically exhibit a higher apparent toughness. The solution to this problem is to use a sufficiently thick specimen to fully contain the plastic zone at the crack tip. The situation is different for SCC. The intergranular SCC associated with nitrate environments is generally thought to propagate by a film rupture mechanism, in which deformation at the crack tip is required. Therefore, the measured $\mathrm{K}_{\text {thSCC }}$ may actually decrease with decreasing constraint.

\section{EPOC Recommendations}

1. Compile all of the fracture mechanics tests run to date on the program and identify discrepancies in the data for the different test techniques.

2. Resolve these discrepancies by means of additional analyses on the specimens and data from previous tests and or additional replicate tests.

3. For the waste chemistries of most interest, run a sufficient number of replicate tests, using the latest test technique, to provide confidence in the $\mathrm{K}_{\text {thSCC }}$ values determined. Note that these tests should be performed after the issue of specimen thickness (Recommendations 4 and 5 ) is resolved.

4. For a few basic chemistries (e.g., 241-AN-107 and $5 \mathrm{M}$ Nitrate), explore the effect of specimen thickness (level of constraint) on $\mathrm{K}_{\mathrm{thSCC}}$.

5. If $\mathrm{K}_{\text {thSCC }}$ is found to be a function of specimen thickness (the level of constraint), perform a stress analysis for the most likely crack geometry in the tank in order to optimize the test specimen thickness.

6. Run $\mathrm{K}_{\text {thSCC }}$ tests of weld and HAZ microstructures for the basic waste chemistries.

\subsection{TANK 241-AN-102 CORROSION PROBE}

The design of the new corrosion probe seems to be much more robust than the previous probe design and addresses the primary flaws in the earlier design. The EPOC discussed the response trigger criteria and frequency of measurements that should be recommended in the future.

\section{Data Quality Objectives}

In order for CH2M-HILL to incorporate the readings from the corrosion probe into routine operations, response trigger criteria values must be identified and incorporated into routine operations. We were asked to assess the data to-date and recommend such "trigger" values. After significant discussion, the EPOC determined that the small amount of data collected from the probe during the relatively short exposure time is insufficient for making such a recommendation. This recommendation was deferred to a later date.

Regarding the ongoing measurements, the decision was made to continue to take the readings manually and at frequencies recommended by the EPOC. For the first six weeks (starting in early 
May) the measurements were taken on a daily basis. For the next six weeks, the frequency was reduced to 3 times per week. Currently, readings are taken once per week.

\section{EPOC Recommendations}

1. After 1 year of data is collected from the 241-AN-102 corrosion probe, the EPOC will be in a position to recommend a path forward to establish data quality objectives

2. The primary electrodes are expected to fail and in order to better ascertain just when they fail, the EPOC recommends that readings continue to be taken on a weekly basis.

3. We suggest that automated data logging be investigated for use on subsequent probes.

\subsection{TANK 241-AY-101 CORROSION PROBE DESIGN CONSIDERATIONS}

The 241-AN-102 Multi-Probe Corrosion Monitoring System (MPCMS) consists of one "fixed" in-tank probe containing primary reference electrodes, secondary reference electrodes, tank material electrodes, electrical resistance (ER) sensors (for measuring uniform corrosion rate), and stressed and unstressed corrosion coupons. Additionally, the system includes four removable probes designed to hold corrosion coupons at various elevations in the tank. The fixed probe is designed to remain in the tank for the duration of service. The removable probes will be removed at intervals to facilitate inspection of the associated coupons for corrosion damage. Removable probes will not be reinserted in the tank following examination.

A major goal for the 241-AN-102 MPCMS is to measure the open-circuit corrosion potentials of both the tank wall and tank steel electrodes mounted at various elevations on the fixed probe. The corrosion potential data from the tank and tank steel electrodes will be compared with laboratory data to assess whether pitting corrosion or SCC of the tank is likely to occur.

The 241-AN-102 MPCMS fixed probe contains one set of electrodes in the saltcake phase and a second set in the supernatant phase.

Each set of electrodes consists of the following

- Two tank material electrodes,

- Three primary reference electrodes (one each: saturated Calomel electrode, silver/silverchloride electrode, and a unique copper sulfate/silver chloride electrode),

- Three secondary reference electrodes (one each: copper, nickel, and silver),

- One set of coupons (three C-rings and three round coupons), and

- One ER sensor.

There also is an ER sensor and a set of coupons in the vapor space on the fixed probe. The fixed probes and removable probes also contain bar shaped coupons that span the LAI.

Up to three MPCMSes will be installed in tanks during FY 2009. The first system has been design, is ready for fabrication, and is based on the 241-AN-102 design, but the copper 
sulfate/silver chloride electrode has been replaced with a copper/copper sulfate electrode. The second MPCMS is currently in the design phase for installation in Tank 241-AY-101. The third planned system would be installed in Tank 241-AN-107, which be based on the design of the 241-AY-102 and 241-AN-102 systems to support the stagnant storage operations in the tank.

The EPOC discussed changes necessary in the Tank 241-AY-101 system because unlike other tanks it MPCMS is currently in the design phase for 241-AY-101. Unlike 241-AN-102, this tank will be used for waste retrieval operations and the supernate and sludge levels in the tank will vary with time. The issue of electrode and coupon type and placement on this new system was discussed at the EPOC meeting. Panel members concluded that the current design of the 241-AN-102 MPCMS (as described above) should be modified for use in 241-AY-101. Specifically, the 241-AY-101 MPCMS should include three "full" sets of electrodes plus three "reduced" sets of electrodes. Electrodes should be placed at elevations designed to collect corrosion data from the present supernatant and sludge levels and expected future supernatant and sludge levels. For the 241-AY-101 MPCMS, a "full" set of electrodes includes:

- Two silver/silver-chloride primary reference electrodes,

- Three secondary reference electrodes (one each: copper, nickel, and silver),

- Four tank material electrodes (two oxidized steel and two bare steel),

- One set of coupons (three c-rings and three round coupons), and

- One ER sensor.

A "reduced" set of electrodes includes:

- Two tank material electrodes (one oxidized and one bare steel), and

- Three secondary reference electrodes (one each: copper, nickel, and silver).

Primary reference electrodes (initially included in the "reduced" set of electrodes) were removed from the "reduced" set since corrosion potential measurements can be made using the primary reference electrodes in the "full" sets of electrodes (three full sets are included on the 241-AY-101 MPCMS design).

Additionally, the 241-AY-101 MPCMS fixed probe should include an ER sensor and a set of coupons in the vapor space, plus a set of bar coupons that span the expected locations of the LAI. Five bar-shaped coupons will be used for this purpose on each of the 241-AY-101 fixed and removable probes. Finally, the EPOC considers that the copper sulfate/silver chloride primary electrode design is faulty and should be eliminated from the probe for 241-AY-101. The saturated Calomel reference electrode has performed well on the 241-AN-102 MPCMS, but overall, the silver-silver chloride reference electrode has performed the best. Thus, only silversilver chloride primary reference electrodes will be used on the 241-AY-101 MPCMS.

\section{EPOC Recommendations}

1. Based on experience with the 241-AN-102 MPCMS, discontinue use of the hybrid copper sulfate/silver chloride primary electrodes and the saturated Calomel primary reference electrodes. 
2. Include additional bar specimens on the fixed and removable probes that span the expected locations of the LAI.

3. Provide additional electrodes, at various levels that could be immersed in the waste or located in the vapor space, based on the anticipated waste additions and removals.

4. Where feasible, provide redundant electrode arrays.

5. Prior to October 1, 2008, the EPOC will review the electrode placement and discuss any revised recommendations with cognizant CH2M-HILL personnel. 